\title{
Equivalent Average Trajectory Dynamics using the Reduced Low-Thrust Coefficients
}

\author{
Jennifer S. Hudson* \\ University of Michigan, Ann Arbor, MI 48019
}

\author{
Daniel J. Scheeres ${ }^{\dagger}$ \\ University of Colorado, Boulder, CO 80309
}

\begin{abstract}
Calculation of low-thrust control laws with equivalent average trajectory dynamics but different thrust profiles is studied using an averaged method based on Fourier series representation of the thrust control. Fourier coefficients of order 2 and higher are used to transform variable-magnitude controls into controls with constant thrust arcs, which can be implemented more easily by low-thrust propulsion systems. Fuel cost reduction through selection of higher-order Fourier coefficients is also discussed.
\end{abstract}

\section{Nomenclature}

$\begin{array}{ll}a & \text { semi-major axis } \\ E & \text { eccentric anomaly } \\ e & \text { eccentricity } \\ \mathbf{F} & \text { thrust acceleration } \\ F_{R} & \text { radial thrust acceleration } \\ F_{S} & \text { normal thrust acceleration } \\ F_{W} & \text { circumferential thrust acceleration } \\ i & \text { inclination } \\ o & \text { any orbital element } \\ M & \text { mean anomaly } \\ n & \text { mean motion } \\ \Omega & \text { longitude of the ascending node } \\ \omega & \text { argument of periapsis } \\ \mu & \text { standard gravitational parameter }\end{array}$

\section{Introduction}

Previous work ${ }^{1,2}$ has demonstrated an averaging method to model low-thrust spacecraft trajectory dynamics using Fourier series representation of the control. Gauss's variational equations were averaged to define a set of secular equations, which are functions of only 14 of the thrust Fourier coefficients, regardless of the order of the original Fourier series. These secular equations can represent thrust controls of varying magnitude and direction, and accurately represent the average trajectory over many spiral orbits. Iterative methods using these averaged secular equations have been shown to solve orbital targeting and optimal control problems.

The current study explores the use of the Fourier coefficients beyond the 14 that appear in the averaged secular equations. These higher-order coefficients do not affect the fundamental trajectory dynamics; thus they can be used to modify a calculated optimal control to meet implementation requirements or further reduce fuel costs without altering the trajectory.

To demonstrate this concept, several methods have been developed to transform a variable-magnitude control into a step control. Most current low-thrust spacecraft engines are able to operate over a range

${ }^{*} \mathrm{PhD}$ Candidate, Department of Aerospace Engineering, University of Michigan

${ }^{\dagger}$ A. Richard Seebass Professor, Aerospace Engineering Sciences, Colorado Center for Astrodynamics Research, University of Colorado. 
of thrust levels, but operators of these systems often prefer to minimize switching between throttle points. Therefore a control based on constant thrust arcs is desirable. This paper will present three methods for selecting higher-order Fourier coefficients to create constant-thrust controls with average trajectory dynamics equivalent to those of variable-thrust controls. Extensions of this method to fuel cost reduction will also be discussed.

\section{Methods}

Previously, we derived the secular equations by averaging the Gauss form of the Lagrange Planetary Equations over one orbit period with respect to mean anomaly. We assumed an acceleration vector with a sufficiently low magnitude that the size and shape of the orbit does not change significantly over one revolution:

$$
\overline{\dot{o}}=\frac{1}{2 \pi} \int_{0}^{2 \pi} \dot{o} d M
$$

Here $o$ represents any orbit element. We resolved the thrust acceleration vector $\overrightarrow{\mathbf{F}}$ along the radial, normal, and circumferential directions and represented each component as a Fourier series expanded in eccentric anomaly,

$$
\begin{aligned}
\overrightarrow{\mathbf{F}} & =F_{R} \hat{\mathbf{r}}+F_{W} \hat{\mathbf{w}}+F_{S}(\hat{\mathbf{w}} \times \hat{\mathbf{r}}) \\
F_{R} & =\sum_{k=0}^{\infty}\left[\alpha_{k}^{R} \cos k E+\beta_{k}^{R} \sin k E\right] \\
F_{W} & =\sum_{k=0}^{\infty}\left[\alpha_{k}^{W} \cos k E+\beta_{k}^{W} \sin k E\right] \\
F_{S} & =\sum_{k=0}^{\infty}\left[\alpha_{k}^{S} \cos k E+\beta_{k}^{S} \sin k E\right],
\end{aligned}
$$

where $\hat{\mathbf{r}}=\frac{\overrightarrow{\mathbf{r}}}{|\overrightarrow{\mathbf{r}}|}$ and $\hat{\mathbf{w}}=\frac{\overrightarrow{\mathbf{r}} \times \overrightarrow{\mathbf{v}}}{|\overrightarrow{\mathbf{r}} \times \overrightarrow{\mathbf{v}}|}$. When these Fourier series for the thrust vector components are substituted into the averaged Gauss equations and the independent parameter for the averaging is shifted to eccentric anomaly, the orthogonality conditions eliminate all but the 0th, 1st, and 2nd order coefficients of each thrust acceleration Fourier series. Thus, the averaged secular Gauss equations are:

$$
\begin{aligned}
\overline{\dot{a}} & =2 \sqrt{\frac{a^{3}}{\mu}}\left[\frac{1}{2} e \beta_{1}^{R}+\sqrt{1-e^{2}} \alpha_{0}^{S}\right] \\
\overline{\dot{e}} & =\sqrt{\frac{a}{\mu}} \sqrt{1-e^{2}}\left[\frac{1}{2} \sqrt{1-e^{2}} \beta_{1}^{R}+\alpha_{1}^{S}-\frac{3}{2} e \alpha_{0}^{S}-\frac{1}{4} e \alpha_{2}^{S}\right] \\
\overline{\dot{i}} & =\sqrt{\frac{a}{\mu}} \frac{1}{\sqrt{1-e^{2}}}\left[\frac{1}{2}\left(1+e^{2}\right) \cos \omega \alpha_{1}^{W}-\frac{3}{2} e \cos \omega \alpha_{0}^{W}-\frac{1}{2} \sqrt{1-e^{2}} \sin \omega \beta_{1}^{W}\right. \\
\overline{\dot{\Omega}} & =\sqrt{\frac{a}{\mu}} \frac{\csc i}{\sqrt{1-e^{2}}}\left[\frac{1}{2} \sqrt{1-e^{2}} \cos \omega \beta_{1}^{W}+\frac{1}{2}\left(1+e^{2}\right) \sin \omega \alpha_{1}^{W}-\frac{3}{2} e \sin \omega \alpha_{0}^{W}\right. \\
\overline{\dot{\omega}}= & \left.-\frac{1}{4} e \sqrt{1-e^{2}} \cos \omega \beta_{2}^{W}-\frac{1}{4} e \sin \omega \alpha_{2}^{W}\right] \\
\overline{\dot{\epsilon}}= & \sqrt{\frac{a}{\mu}} \frac{1}{\mu}\left[-\frac{1}{2} \sqrt{1-e^{2}} \alpha_{1}^{R}+e \sqrt{1-e^{2}} \alpha_{0}^{R}+\frac{1}{2}\left(2-e^{2}\right) \beta_{1}^{S}-\frac{1}{4} e \beta_{2}^{S}\right]-\cos i \dot{\Omega} \\
& \left(1-\sqrt{1-e^{2}}\right)(\overline{\dot{\omega}}+\overline{\dot{\Omega}})+2 \sqrt{1-e^{2}} \sin ^{2}\left(\frac{i}{2}\right) \overline{\dot{\Omega}} .
\end{aligned}
$$


These equations are functions of the 14 coefficients

$$
\vec{\alpha}=\left[\begin{array}{c}
\alpha_{0}^{R} \\
\alpha_{1}^{R} \\
\alpha_{2}^{R} \\
\beta_{1}^{R} \\
\alpha_{0}^{S} \\
\alpha_{1}^{S} \\
\alpha_{2}^{S} \\
\beta_{1}^{S} \\
\beta_{2}^{S} \\
\alpha_{0}^{W} \\
\alpha_{1}^{W} \\
\alpha_{2}^{W} \\
\beta_{1}^{W} \\
\beta_{2}^{W}
\end{array}\right]
$$

These secular equations can be used to efficiently solve orbital targeting problems using an iterative method, as described previously. ${ }^{2}$ We minimze a cost function while solving for a set of 14 coefficients that control the trajectory to meet the targeting objectives. Any cost function that is a function of the thrust acceleration may be used, but we often prefer the form

$$
J(\vec{\alpha})=\frac{1}{2 \pi} \int_{0}^{2 \pi}\left(F_{R}^{2}+F_{S}^{2}+F_{W}^{2}\right) d M,
$$

which represents the minimum energy solution. This cost function allows us to analytically evaluate the necessary partial derivatives to iteratively update the control coefficients in Equation 12. Targeting methods using this approach have been shown to efficiently and accurately solve orbital targeting problems over many spiral orbits.

\section{Coefficient Selection}

Only the 14 coefficients of the thrust acceleration Fourier series shown in Equation 12 control the average trajectory dynamics of the low-thrust spacecraft. However, the other coefficients of order 3 and higher can still affect the shape and frequency of the control itself. They may be set to zero, or they may be selected to shape the control into a more desirable form without altering the average trajectory.

In this paper we explore several approaches for selecting these higher-order coefficients. First, they may be selected to reduce the fuel or energy cost of a transfer. An existence proof of this cost-reduction potential has previously been developed. ${ }^{3}$

The coefficients may also be selected to transform the control into a form that is more easily implemented by existing low-thrust engines. Current electric propulsion systems operate on a range of fixed throttle points, and minimal switching is preferred. Thus it is desirable to transform our continuously-varying controls into step functions that produce equivalent average trajectory dynamics. In some cases, the magnitude of the total thrust acceleration within the step function can also be made constant, to mimic the design of a typical spacecraft with one gimbaled low-thrust engine.

\section{A. Existence of Equivalent, Lower-Cost Control}

Previously, ${ }^{3}$ we have shown that Fourier coefficients beyond the 14 that appear in the secular equations can reduce the cost of a transfer without affecting the trajectory. For example, if the $\alpha_{2}$ coefficient for any of the three directional Fourier series is nonzero, the higher order $\alpha$ coefficients of that direction can be chosen according to Equation 14,

$$
\alpha_{n}=(e \epsilon)^{n-2} \alpha_{2}
$$


where $e$ is eccentricity and $0<\epsilon<1$. If $n \rightarrow \infty$, the reduction in the minimum energy cost function (Equation 13) is

$$
\Delta J=-\frac{1}{2} \alpha_{2}^{2} \frac{e^{2}}{1-(e \epsilon)^{2}}(1-\epsilon) .
$$

In practice, this method generally results in very small reductions in total energy cost. Other methods may be developed to improve or optimize the coefficient selection methodology for greater cost reductions.

\section{B. Equivalent Control Function: One Step}

The higher-order thrust acceleration Fourier coefficients may also be used to shape the control function for improved implementation. There are many possible ways to perform this shaping transformation. As a simple case for our initial analysis, we choose a function shape with only one step per orbit.

Assume the 14 relevant Fourier coefficients of a control have been selected to achieve a given orbit transfer. We may then select additional coefficients to make the control take the form of a step function. We begin by considering the step function pictured in Figure 1.

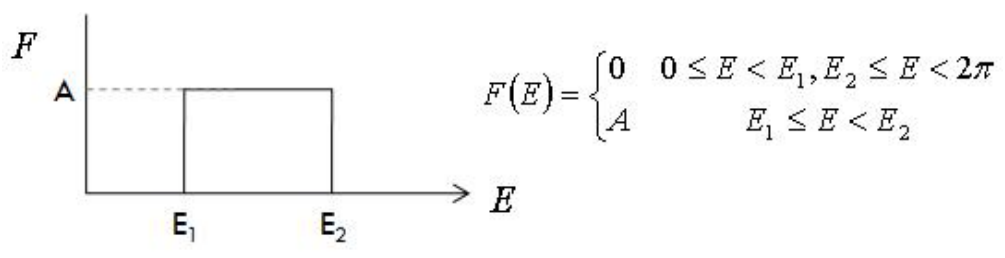

Figure 1. Step Tangential Acceleration

This function is defined by three parameters: $A, E_{1}$, and $E_{2}$, or equivalently, $A, \Delta E=E_{2}-E_{1}$, and $\bar{E}=\frac{E_{1}+E_{2}}{2}$. The Fourier series for this function is defined by equations 16-19.

$$
\begin{aligned}
F & =\sum_{k=0}^{\infty} \alpha_{k} \cos (k E)+\beta_{k} \sin (k E) \\
\alpha_{0} & =\frac{1}{2 \pi} \int_{E_{1}}^{E_{2}} A d E \\
\alpha_{k} & =\frac{1}{\pi} \int_{E_{1}}^{E_{2}} A \cos (k E) d E \\
\beta_{k} & =\frac{1}{\pi} \int_{E_{1}}^{E_{2}} A \sin (k E) d E
\end{aligned}
$$

The 0th, 1st, and 2nd order coefficients are fixed by our targeting requirements. For simplicity in our initial analysis, we consider only the 0th and 1st order coefficients in a single-direction force:

$$
\begin{aligned}
& \alpha_{0}=\frac{1}{2 \pi} \int_{E_{1}}^{E_{2}} A d E=\frac{A}{2 \pi} \Delta E \\
& \alpha_{1}=\frac{1}{\pi} \int_{E_{1}}^{E_{2}} A \cos (k E) d E=\frac{2 A}{\pi} \cos \bar{E} \sin \left(\frac{\Delta E}{2}\right) \\
& \beta_{1}=\frac{1}{\pi} \int_{E_{1}}^{E_{2}} A \sin (k E) d E=\frac{2 A}{\pi} \sin \bar{E} \sin \left(\frac{\Delta E}{2}\right) .
\end{aligned}
$$

We can re-write these equations,

$$
\begin{array}{r}
\bar{E}=\tan ^{-1}\left(\frac{\beta_{1}}{\alpha_{1}}\right) \\
\alpha_{1}^{2}+\beta_{1}^{2}=\left(\frac{2 A}{\pi}\right)^{2} \sin ^{2}\left(\frac{\Delta E}{2}\right) \\
\alpha_{0}=\frac{A}{2 \pi} \Delta E
\end{array}
$$


and find that Equations 24 and 25 lead to the following equation:

$$
\Delta E-\frac{4 \alpha_{0}}{\sqrt{\alpha_{1}^{2}+\beta_{1}^{2}}} \sin \left(\frac{\Delta E}{2}\right)=0 .
$$

Equation 26 is plotted in Figure 2 for various values of the coefficient $c=-\frac{4 \alpha_{0}}{\sqrt{\alpha_{1}^{2}+\beta_{1}^{2}}}$. For a solution to exist, $c$ must be less than approximately -2 .

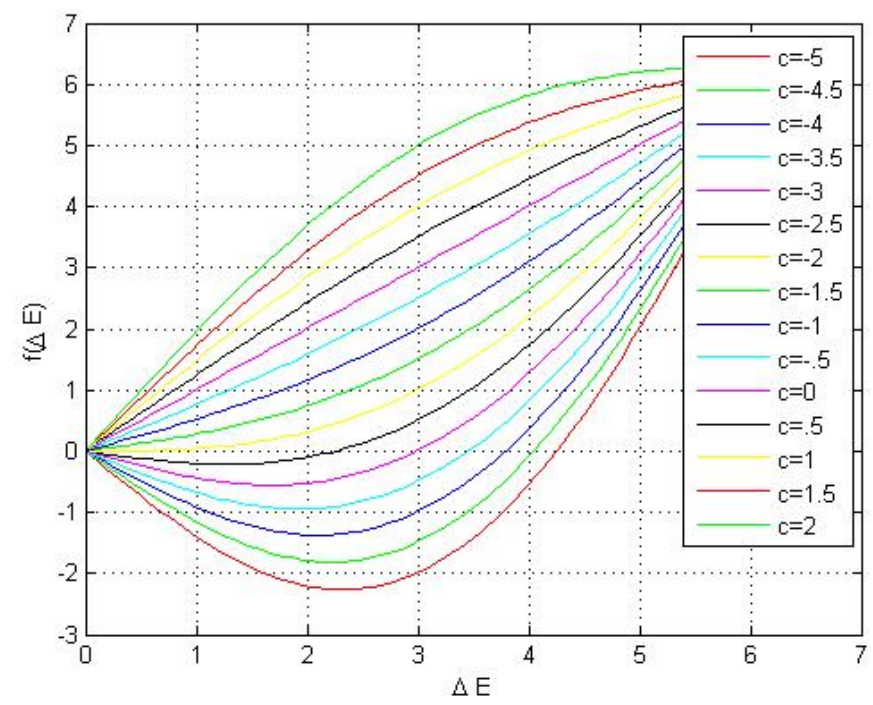

Figure 2. Equation 26, plotted for a range of values of $c$

Assuming the values of $\alpha_{0}, \alpha_{1}$, and $\beta_{1}$ from the targeting control are such that $c<-2$, we can solve for $A, \Delta E$, and $\bar{E}$ and use these values in Equations 17-19 to generate higher-order coefficients for the force Fourier series.

Figures 3 and 4 show an example of this method. In this example, the Fourier coefficients for the original force function were chosen to make $c<-2$, and the resulting trajectory was determined using both the Newtonian equations of motion and averaged secular equations (plotted in blue and red, respectively). Then, the above approach was used to re-calculate Fourier coefficients from order 2 to 100 and the resulting trajectories were again determined using both the Newtonian and averaged secular equations (plotted in black and green, respectively). As shown in Figure 3, the two different controls accomplish the same targeting objectives. 

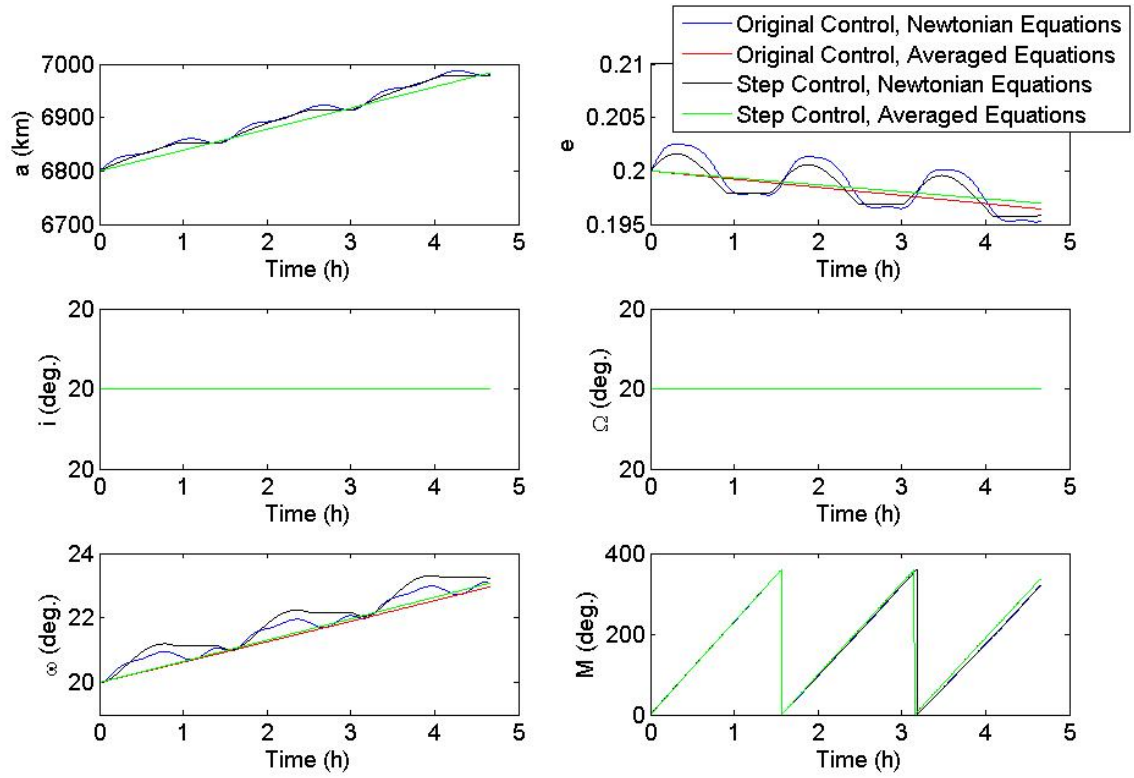

Figure 3. Trajectories due to "equivalent" initial and step thrust acceleration

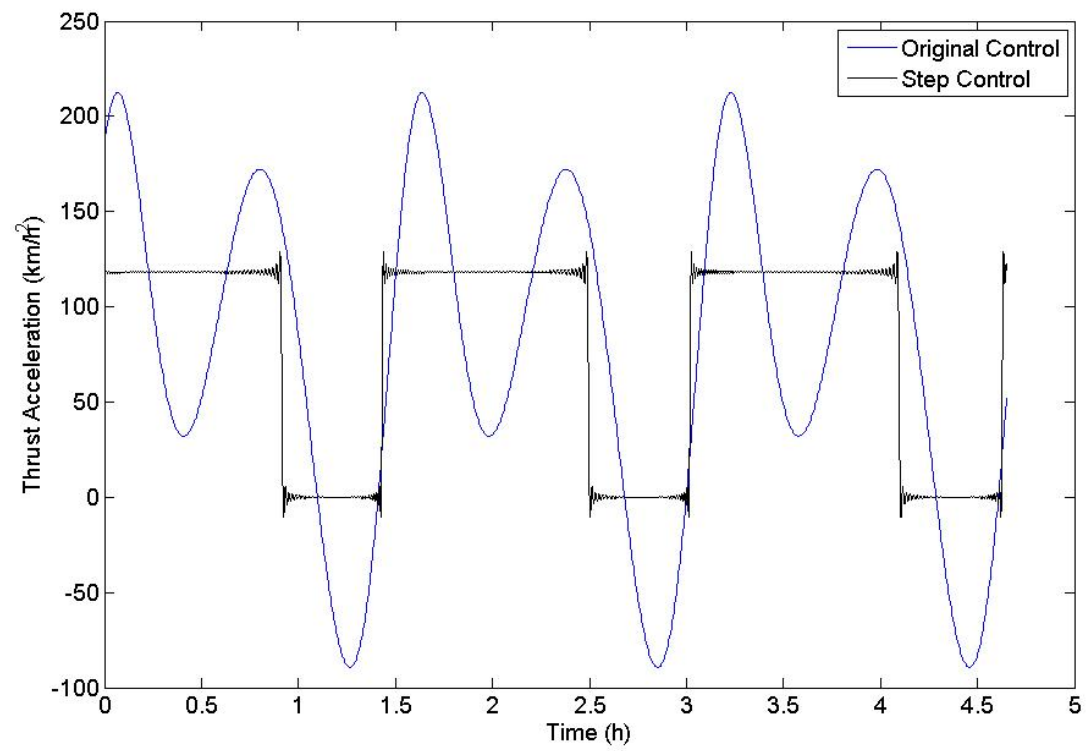

Figure 4. Initial continuously-varying thrust acceleration and its "equivalent" step acceleration 
This method is effective, but its applicability is limited by the $c<-2$ restriction. Also, the significant coefficients $\alpha_{2}$ and $\beta_{2}$ are neglected, which may cause disparities between the trajectories due to the original and transformed controls, particularly over long time spans. (This re-calculating of $\alpha_{2}$ and $\beta_{2}$ is responsible for the difference between the red and green plots in Figure 3).

\section{Equivalent Control Function: Two Steps}

To avoid the limitations and inaccuracies of the one-step approach, we consider a step function shape with five defining parameters, to match the maximum number of significant coefficients in each of the force directions. The function in Figure 5 is defined by $A, E_{1}, E_{2}, E_{3}$, and $E_{4}$ or, equivalently, $A, \Delta E_{1}=E_{2}-E_{1}$, $\bar{E}_{1}=\frac{E_{1}+E_{2}}{2}, \Delta E_{2}=E_{4}-E_{3}, \bar{E}_{2}=\frac{E_{3}+E_{2}}{4}$.

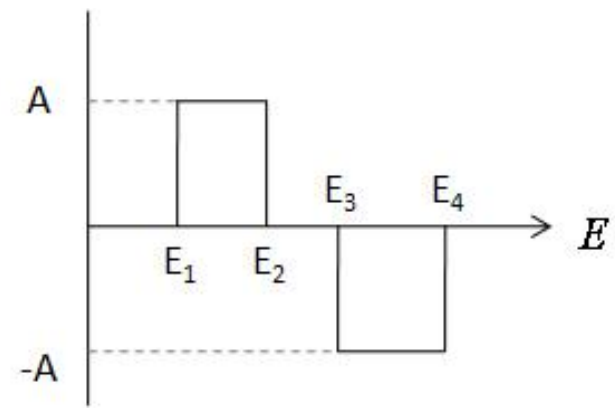

Figure 5. Two steps of same magnitude (opposite sign) and different duration

As above, the 0th, 1st, and 2nd order Fourier coefficients for this function are fixed by the original control:

$$
\begin{aligned}
\alpha_{0} & =\frac{A}{2 \pi}\left(\Delta E_{1}-\Delta E_{2}\right) \\
\alpha_{1} & =\frac{2 A}{\pi}\left(\cos \bar{E}_{1} \sin \frac{\Delta E_{1}}{2}-\cos \bar{E}_{2} \sin \frac{\Delta E_{2}}{2}\right) \\
\beta_{1} & =\frac{2 A}{\pi}\left(\sin \bar{E}_{1} \sin \frac{\Delta E_{1}}{2}-\sin \bar{E}_{2} \sin \frac{\Delta E_{2}}{2}\right) \\
\alpha_{2} & ==\frac{A}{\pi}\left(\cos 2 \bar{E}_{1} \sin \Delta E_{1}-\cos 2 \bar{E}_{2} \sin \Delta E_{2}\right) \\
\beta_{2} & =\frac{A}{\pi}\left(\sin 2 \bar{E}_{1} \sin \Delta E_{1}-\sin 2 \bar{E}_{2} \sin \Delta E_{2}\right) .
\end{aligned}
$$

By definition, there are several constraints on the angles, which can be used to find constraints between the coefficients and the amplitude,

$$
\begin{array}{r}
0 \leq \Delta E_{i} \leq 2 \pi \\
0 \leq \bar{E}_{i} \leq 2 \pi \\
-2 \pi \leq \Delta E_{1}-\Delta E_{2} \leq 2 \pi,
\end{array}
$$

where $i=1,2$. From these, and allowing $A$ to be positive or negative, the following limits can be placed on the coefficients:

$$
\begin{array}{r}
-|A| \leq \alpha_{0} \leq|A| \\
-|A| \leq \frac{\pi \alpha_{1}}{4} \leq|A| \\
-|A| \leq \frac{\pi \beta_{1}}{4} \leq|A| \\
-|A| \leq \frac{\pi \alpha_{2}}{2} \leq|A| \\
-|A| \leq \frac{\pi \beta_{2}}{2} \leq|A| .
\end{array}
$$


These relations dictate a minimum magnitude of $A$ in order for real solutions to exist. To solve Equations 27-31 using a numerical method, a good starting guess is $A=\max \left\{\alpha_{0}, \frac{\pi \alpha_{1}}{4}, \frac{\pi \beta_{1}}{4}, \frac{\pi \alpha_{2}}{2}, \frac{\pi \beta_{2}}{2}\right\}$ or $A=$ $\min \left\{\alpha_{0}, \frac{\pi \alpha_{1}}{4}, \frac{\pi \beta_{1}}{4}, \frac{\pi \alpha_{2}}{2}, \frac{\pi \beta_{2}}{2}\right\}$, whichever is largest in magnitude.

As the magnitude of $A$ increases beyond this minimum, the relative sizes of the pulses must decrease, according to Equation 27. The following relations may also be useful in choosing initial guesses for the unknown parameters:

$$
\begin{aligned}
& \alpha_{1}^{2}+\beta_{1}^{2}=\left(\frac{2 A}{\pi}\right)^{2}\left[\sin ^{2} \frac{\Delta E_{1}}{2}+\sin ^{2} \frac{\Delta E_{2}}{2}-2 \cos \left(\bar{E}_{1}-\bar{E}_{2}\right) \sin \frac{\Delta E_{1}}{2} \sin \frac{\Delta E_{2}}{2}\right] \\
& \alpha_{2}^{2}+\beta_{2}^{2}=\left(\frac{A}{\pi}\right)^{2}\left[\sin ^{2} \Delta E_{1}+\sin ^{2} \Delta E_{2}-2 \cos 2\left(\bar{E}_{1}-\bar{E}_{2}\right) \sin \frac{\Delta E_{1}}{2} \sin \frac{\Delta E_{2}}{2}\right] .
\end{aligned}
$$

Figures 6 - 9 show an example of this method. The initial control was the solution to a targeting problem in which all six orbital elements were changed over three revolutions. Each directional component of the control was transformed into a two-step control with equivalent average trajectory dynamics. Matlab's fsolve function was used to solve Equations 27 - 31, initialized with guesses based on the relations described above.
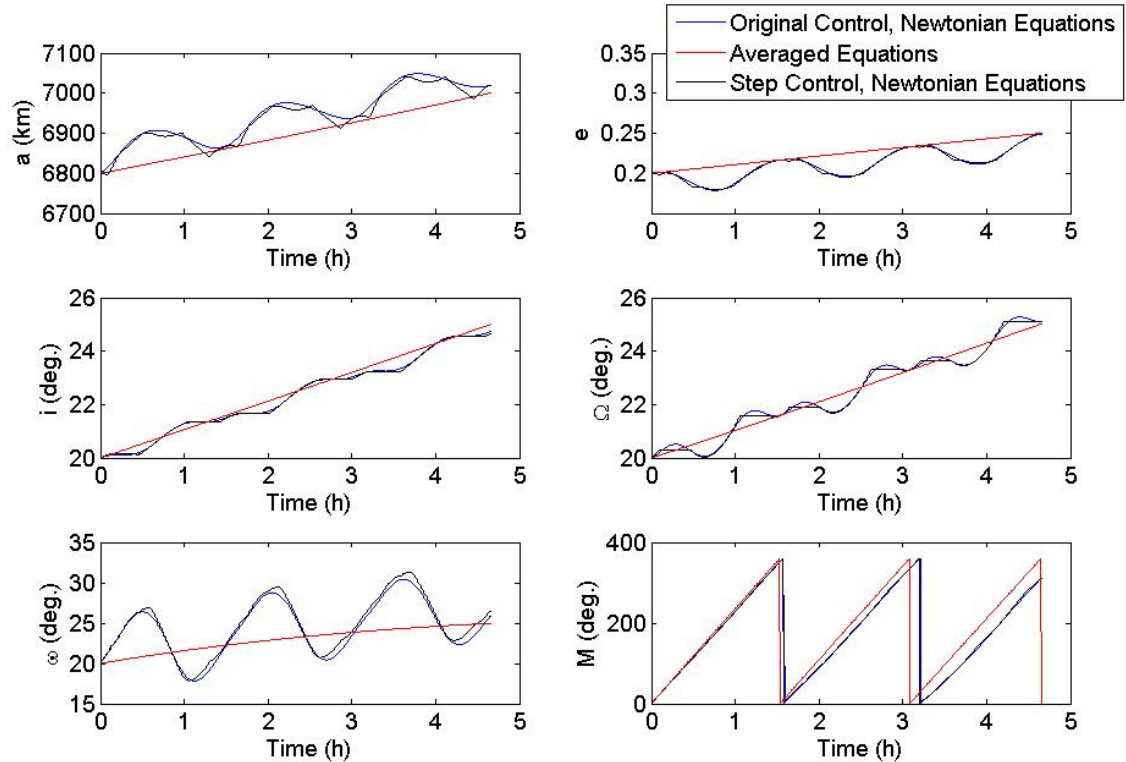

Figure 6. Trajectories due to "equivalent" inital and two-step controls 


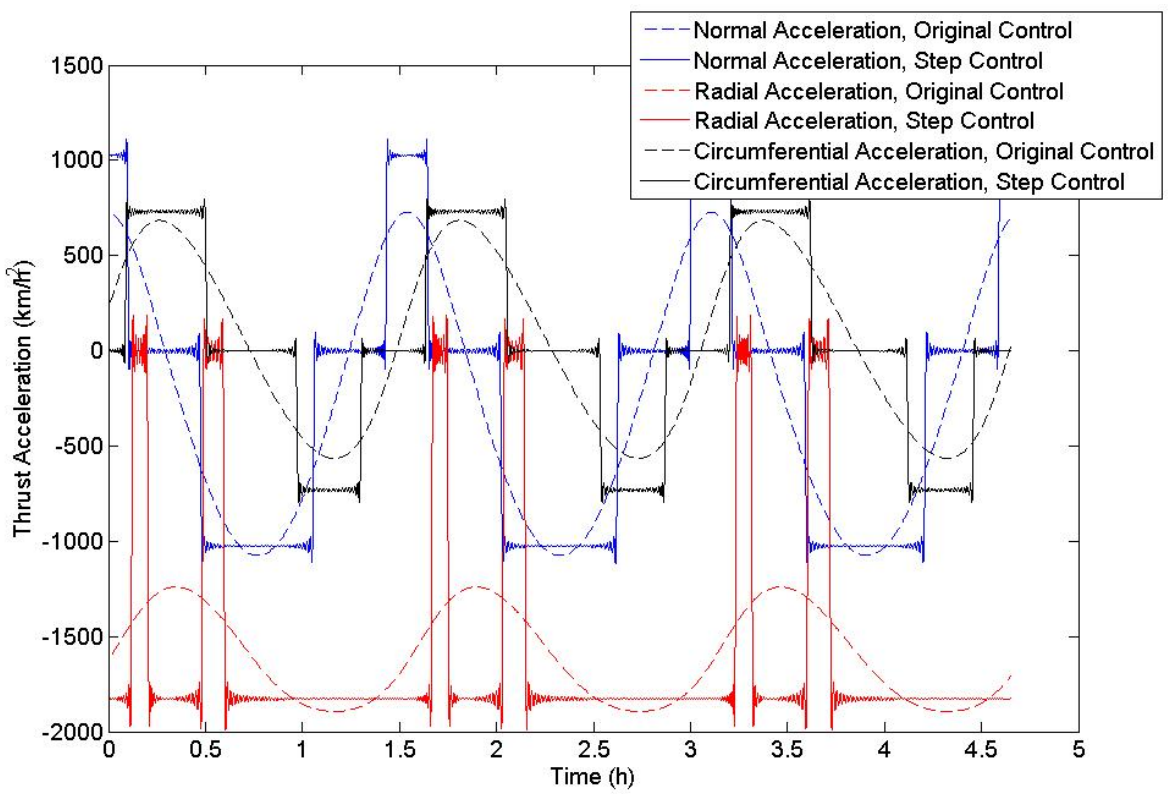

Figure 7. Thrust Acceleration vs. Time

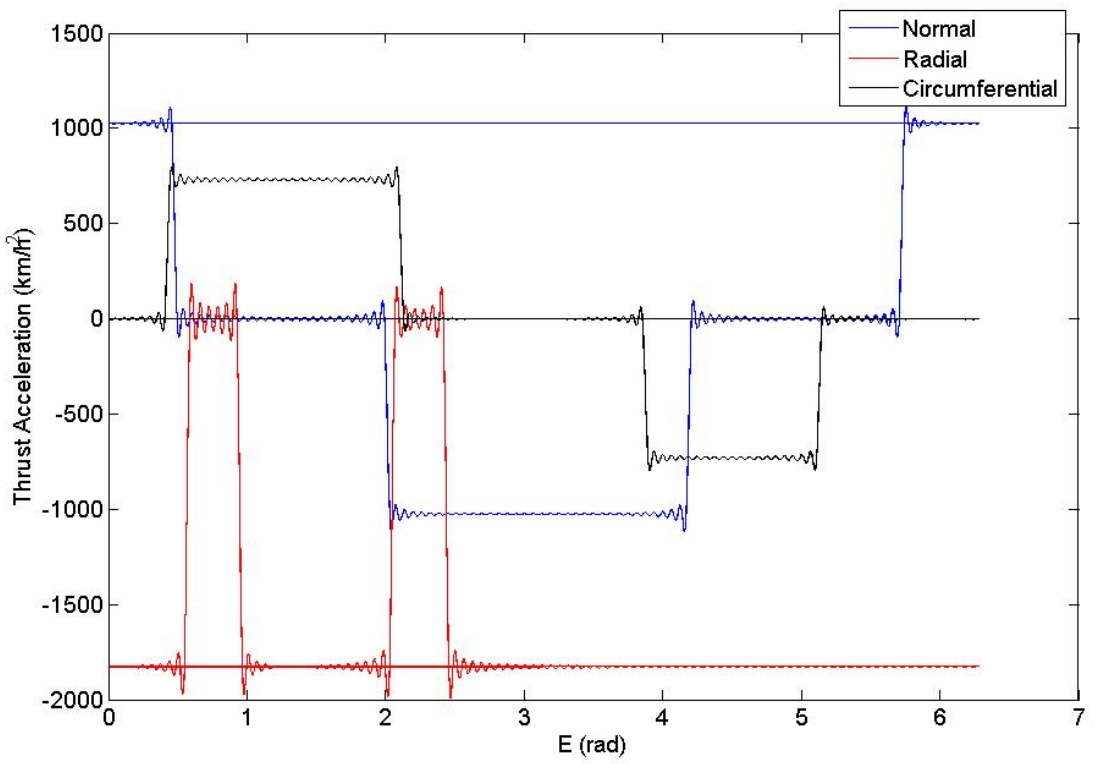

Figure 8. Thrust Acceleration vs. Eccentric Anomaly 


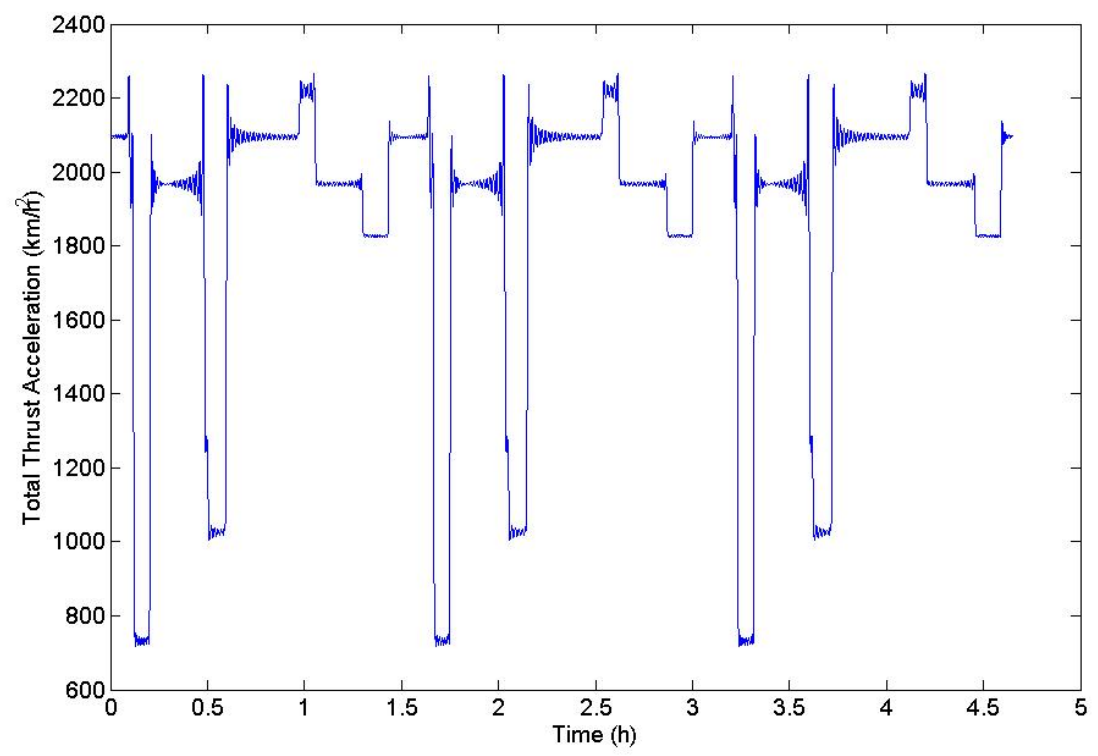

Figure 9. Total Thrust Acceleration $\sqrt{F_{W}^{2}+F_{R}^{2}+F_{S}^{2}}$

This approach effectively transforms each directional component of the control into an "equivalent" step control. The velocity increment, $\Delta V$, for the orbit transfer with the original control was $2320.6 \mathrm{~m} / \mathrm{s}$. The $\Delta V$ for the transfer with the step control was $2436.5 \mathrm{~m} / \mathrm{s}$, a $5 \%$ increase. Simulations indicate that transformation to a step control in this manner usually results in a decrease or small increase in $\Delta V$.

Solutions have been found to exist for most cases when the initial control is the solution to a targeting problem. In some cases, multiple solutions for $A, \bar{E}_{1,2}$, and $\Delta E_{1,2}$ can be found, although these different solution sets usually describe the same step function.

\section{Constant-Magnitude Control}

These transformations to equivalent controls are motivated by the operational profiles of low-thrust propulsion systems. The method described in the previous section transforms each directional component of the control to a step function, which is a valuable improvement, but does not consider the relations between the directional components. As shown in Figure 9, this can result in a lot of switching for the spacecraft engine, which must change its thrust direction and magnitude at each increment.

A more applicable approach should consider the control for all thrust directions simultaneously. We consider a control made up of three steps of constant magnitude, as pictured in Figure 10. The direction of the force, $\lambda$, stays constant throughout the step, but may be different for each step. To simplify the calculations, we consider the case of planar thrust only, as shown in Figure 11. 


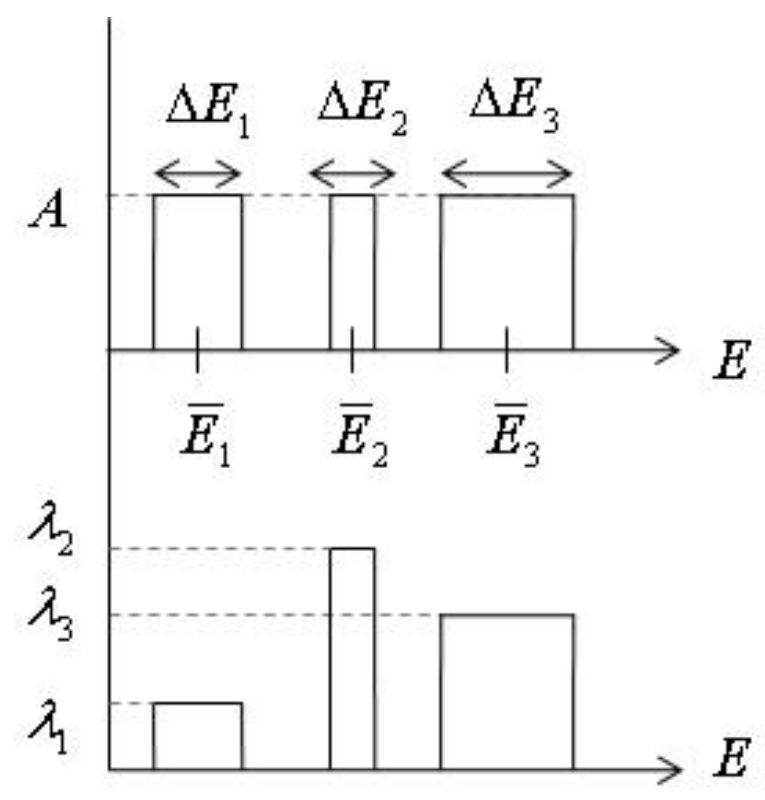

Figure 10. Three steps of constant magnitude, varying direction and duration

This thrust acceleration profile has 10 unknown parameters: $A, \bar{E}_{1}, \bar{E}_{2}, \bar{E}_{3}, \Delta E_{1}, \Delta E_{2}, \Delta E_{3}, \lambda_{1}, \lambda_{2}$, and $\lambda_{3}$.

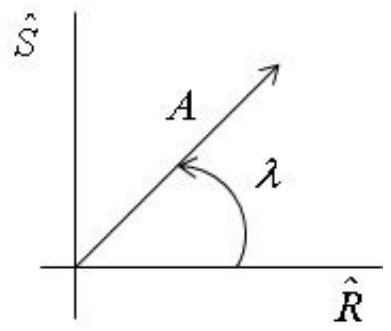

Figure 11. Planar Acceleration

Using the definitions of Fourier coefficients, we can write equations for the nine key acceleration coefficients in terms of these unknown parameters (the tenth coefficient, $\alpha_{2}^{R}$, does not appear in the averaged equations, so we set it to zero). We solve for the unknowns, then use them to calculate higher-order Fourier coefficients to transform the control into step form.

$$
\begin{aligned}
\alpha_{0}^{R} & =\frac{A}{2 \pi} \sum_{i=1}^{3}\left(\cos \left(\lambda_{i}\right) \Delta E_{i}\right) \\
\alpha_{k}^{R} & =\frac{2 A}{k \pi} \sum_{i=1}^{3}\left(\cos \left(\lambda_{i}\right) \cos \left(k \bar{E}_{i}\right) \sin \left(\frac{k \Delta E_{i}}{2}\right)\right) \\
\beta_{k}^{R} & =\frac{2 A}{k \pi} \sum_{i=1}^{3}\left(\cos \left(\lambda_{i}\right) \sin \left(k \bar{E}_{i}\right) \sin \left(\frac{k \Delta E_{i}}{2}\right)\right) \\
\alpha_{0}^{S} & =\frac{A}{2 \pi} \sum_{i=1}^{3}\left(\sin \left(\lambda_{i}\right) \Delta E_{i}\right) \\
\alpha_{k}^{S} & =\frac{2 A}{k \pi} \sum_{i=1}^{3}\left(\sin \left(\lambda_{i}\right) \cos \left(k \bar{E}_{i}\right) \sin \left(\frac{k \Delta E_{i}}{2}\right)\right)
\end{aligned}
$$




$$
\beta_{k}^{s}=\frac{2 A}{k \pi} \sum_{i=1}^{3}\left(\sin \left(\lambda_{i}\right) \sin \left(k \bar{E}_{i}\right) \sin \left(\frac{k \Delta E_{i}}{2}\right)\right)
$$

An example of this method is shown below. The initial control was the solution to a targeting problem in which semi-major axis was increased and eccentricity was decreased over three orbits while the other orbital elements were held constant. Table 1 shows the parameters of the solution step function. Figures $12-14$ show the implementation of this solution.

Table 1. Parameters of Equivalent Constant-Magnitude Step Function

\begin{tabular}{cccc}
\hline$A\left(m / s^{2}\right)$ & 382.3 & $\Delta E_{2}($ deg. $)$ & 59.8 \\
$\bar{E}_{1}($ deg. $)$ & 114.3 & $\Delta E_{3}($ deg. $)$ & 62.1 \\
$\bar{E}_{2}$ (deg. $)$ & 247.8 & $\lambda_{1}($ deg. $)$ & 167.3 \\
$\bar{E}_{3}$ (deg. $)$ & 337.4 & $\lambda_{2}$ (deg.) & 185.6 \\
$\Delta E_{1}$ (deg. $)$ & 181.8 & $\lambda_{3}$ (deg. $)$ & 186.7 \\
\hline
\end{tabular}

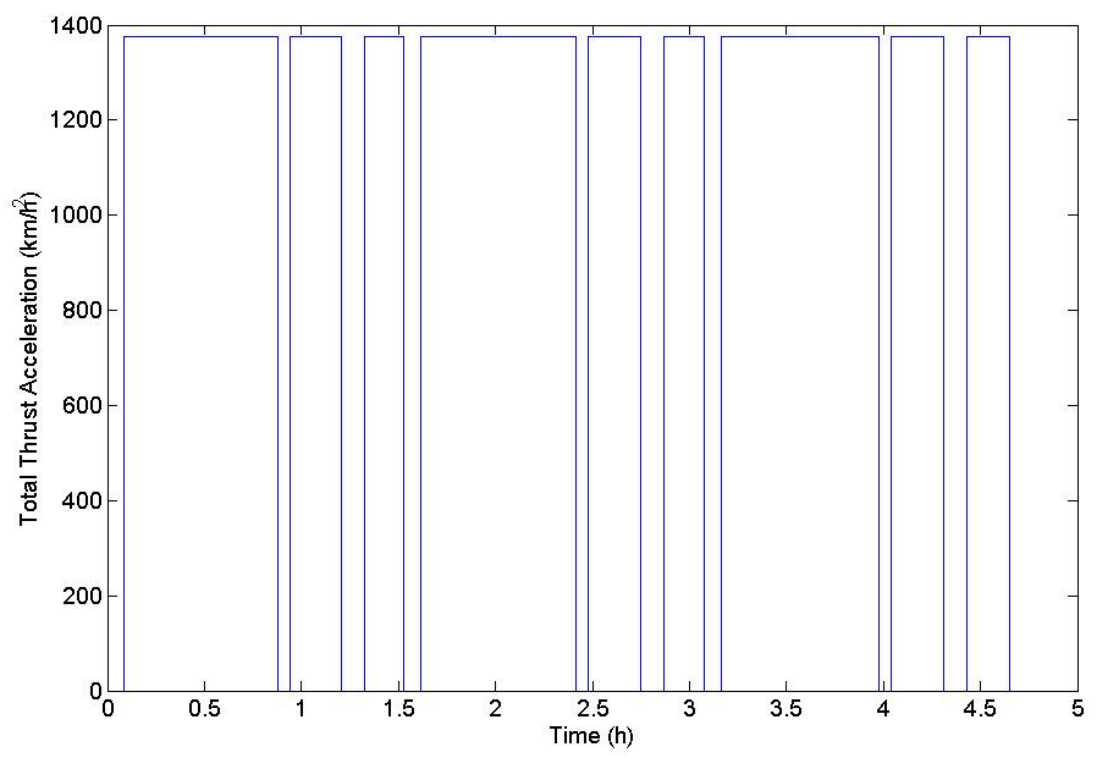

Figure 12. Total Thrust Acceleration 

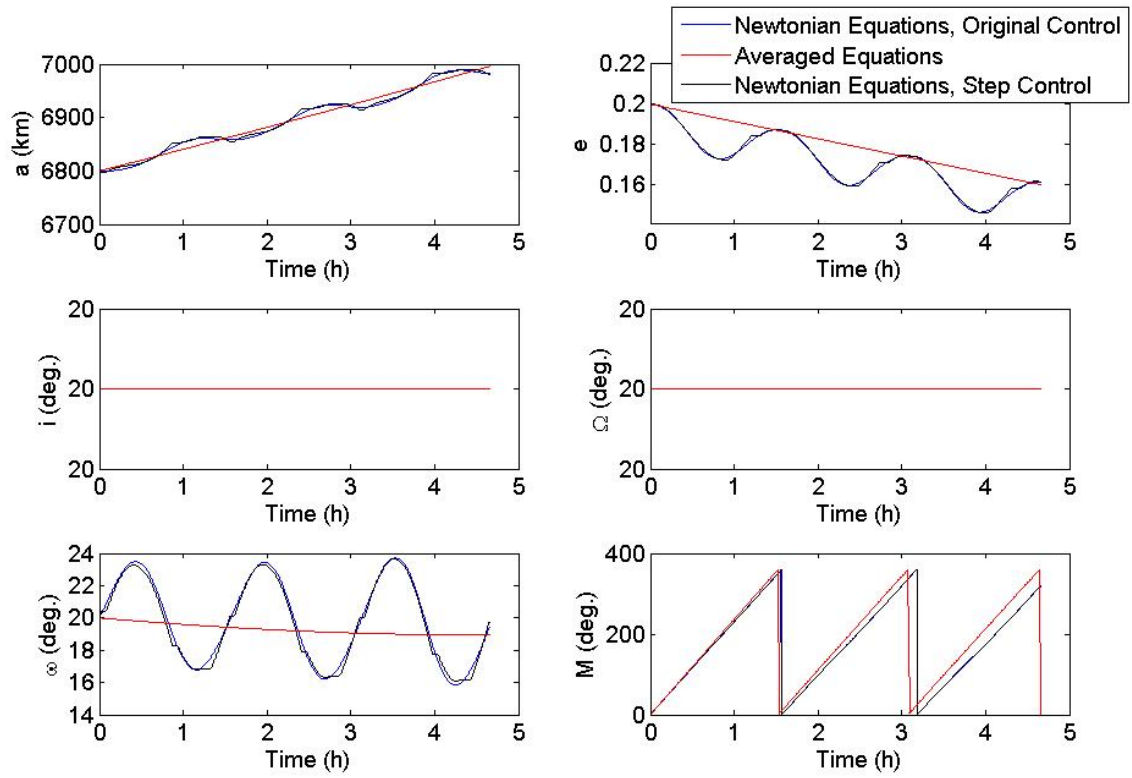

Figure 13. Spacecraft Trajectory

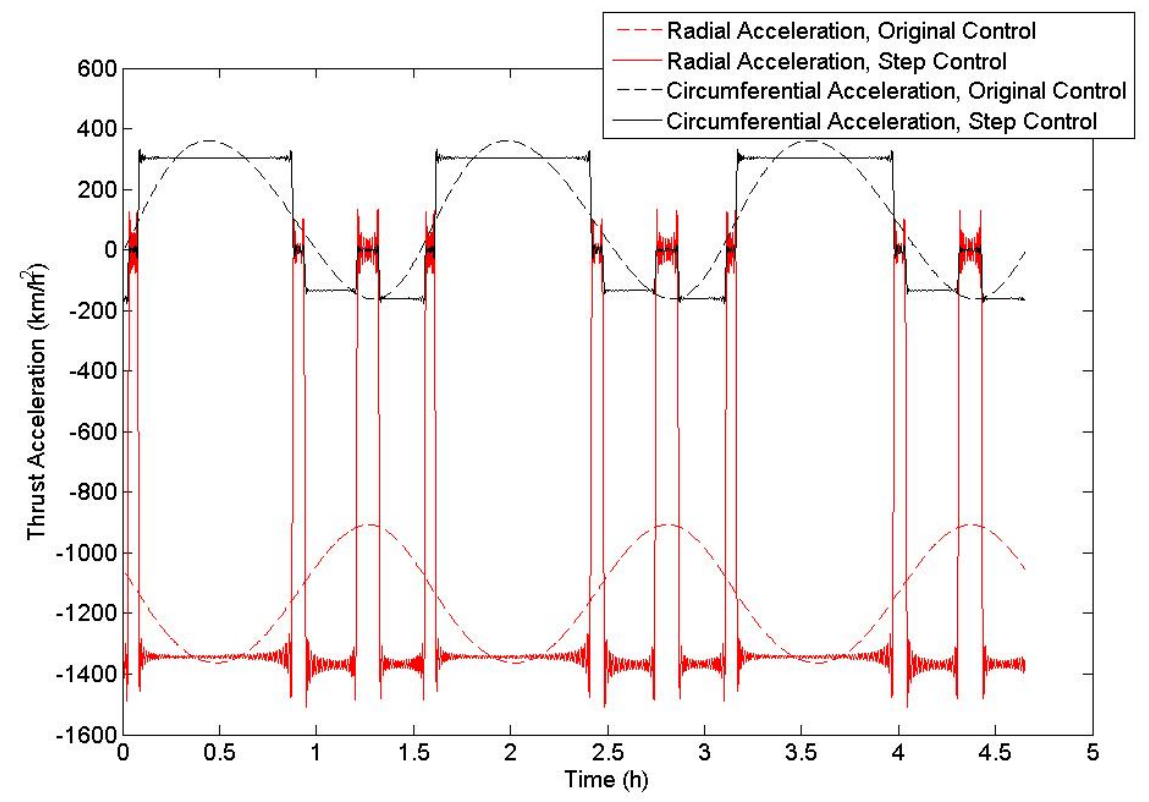

Figure 14. Thrust Acceleration vs. Time 
The velocity increment, $\Delta V$, for the orbit transfer with the original control was $1503.8 \mathrm{~m} / \mathrm{s}$. The $\Delta V$ for the transfer with the constant-magnitude step control was $1515.6 \mathrm{~m} / \mathrm{s}$, a $0.79 \%$ increase.

To make this method fully general, out-of-plane thrust acceleration should be included. This would require a step control defined by 14 parameters with a single acceleration magnitude and variable thrust direction in three dimensions. Any function shape that meets these criteria could be used. For example, four steps of the same magnitude and duration and different angles, as shown in Figure 15, could be defined by the 14 parameters $A, E_{1}, E_{2}, E_{3}, E_{4}, \Delta E, \lambda_{1}, \lambda_{2}, \lambda_{3}, \lambda_{4}, \phi_{1}, \phi_{2}, \phi_{3}$, and $\phi_{4}$.

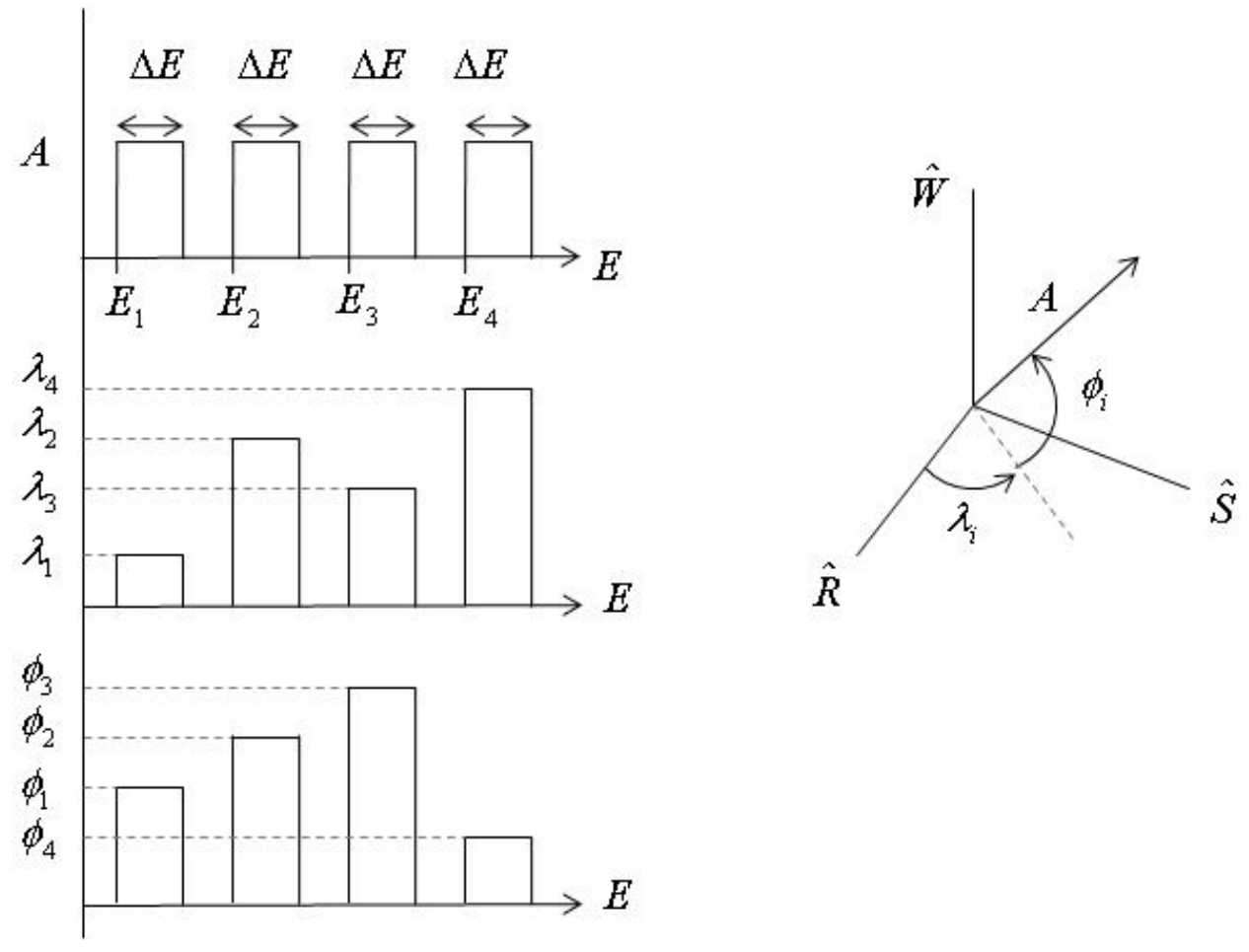

Figure 15. Four steps of constant magnitude and duration, varying direction in 3D 
To shape the original control into this form, we would write the equations for the 14 key coefficients in terms of these 14 unknown parameters, solve for the unknowns, then use them to calculate higher-order Fourier coefficients of the thrust acceleration. The problem of 14 simultaneous equations is challenging, but solutions should exist for some cases. Future work will consider which types of problems can be solved in this manner.

\section{Conclusions}

A general, variable-magnitude, low-thrust control can be transformed into various other types of controls by selection of Fourier coefficients of order 2 or 3 and above. The transformed controls lead to spacecraft trajectories with equivalent average trajectory dynamics.

These transformations can reduce the energy cost of an orbit transfer. They can also reduce the amount of throttling required by the low-thrust engine, by shaping the control into a step function. In some cases, the magnitude of the steps may be set constant, such that the control is simply a set of on/off times and thrust directions.

Numerical examples have shown that transformation to equivalent step controls can increase or decrease the velocity increment required for a given orbit transfer. Future work will explore the mathematical basis for these changes in $\Delta V$, to determine cases and methods in which both shaping and cost reduction can be achieved.

\section{Acknowledgments}

This material is based upon work supported under a National Science Foundation Graduate Research Fellowship.

\section{References}

\footnotetext{
${ }^{1}$ Hudson, J. and Scheeres, D. "Reduction of Low Thrust Continuous Controls for Trajectory Dynamics." Journal of Guidance, Control, and Dynamics, Vol.32, No.3, pp.780-787, 2009.

${ }^{2}$ Hudson, J. and Scheeres, D. "Determination of Fundamental Low-Thrust Control Frequencies for Fitting Sequences of Orbital States." 2010 AAS/AIAA Space Flight Mechanics Meeting, San Diego, CA, February 2010.

${ }^{3}$ Hudson, J. and Scheeres, D. "Trajectory Optimization using the Reduced Eccentric Anonaly Low-Thrust Coefficients." 2008 AIAA/AAS Astrodynamics Specialist Conference, Honolulu, HI, August 2008.
} 